Ann. Génét. Sél. anim., r973, 5 (I), I25-I33.

\title{
VARIATION SUIVANT LA POPULATION DU RAPPORT DE LA VARIANCE DES MALES A CELLE DES FEMELLES POUR LE POIDS CORPOREL CHEZ LA POULE ( $\left.{ }^{(}\right)$
}

\author{
P. MÉRAT et H. AYOUB * \\ Laboratoire de Génétique factorielle, \\ Centre national de Recherches zootechniques, I. N.R. A., \\ 78350 Jouy en Josas \\ * Faculté d'A griculture \\ Université Ain-Shams \\ Le Caire (Égypte)

\section{RÉSUMÉ}

Des différences du rapport de la variance des femelles à celles des mâles pour le poids à divers âges ont été observées entre plusieurs populations de volailles élevées dans les mêmes conditions et dont les poids moyens étaient du même ordre. Une origine génétique de ces différences est vraisemblable.

\section{IN'TRODUC'TION}

Une variation entre populations ou entre familles du rapport des poids moyens à un âge donné dans les deux sexes a été plusieurs fois mise en évidence chez la poule. Un article séparé (Ayoub et MÉRAT, I972) contient une brève revue des travaux faits dans ce sens.

$A$ priori, cependant, c'est non seulement pour la moyenne des poids, mais aussi pour sa variance, que le rapport entre sexes peut varier suivant le groupe génétique considéré. Les données présentes illustrent cette possibilité, non examinée auparavant à notre connaissance.

\section{MATÉRIEL}

Le matériel étudié comprend, en ce qui concerne les poids à 8 semaines, trois populations élevées en 1963 à Jouy en Josas, et, pour le poids à l'âge adulte (Io mois environ), 5 souches de type "ponte " élevées au domaine du Magneraud en 1968.

(1) Cf. également Ayoub et Mérat, r972, Ann. Génét. Sél. anim., 4, 45-49.

Annales de Génétique animale. - I973. 
Nous avons également comparé le rapport de la variance dans les deux sexes pour les poids à 6 et à $\mathbf{I} 2$ semaines, dans une population dérivée de Leghorns (" $\mathrm{L}_{22}$ "), une autre dérivée principalement de Rhode-Island (" $\mathrm{M}_{55}$ 》) et leurs croisements réciproques, élevés en I968 à la Station de Recherches Avicoles à Nouzilly.

Tous les animaux étaient élevés au sol pour les périodes considérées, et dans des conditions identiques à l'intérieur de chaque comparaison ( $\left.{ }^{1}\right)$. Toutefois, chaque souche était élevée dans un local distinct en ce qui concerne les données de Jouy en Josas et du Magneraud.

Les populations du Magneraud comportaient une proportion de mâles gardés jusqu'à l'âge adulte de l'ordre de $30 \mathrm{p}$. Ioo en moyenne. Par contre, aucun tri n'était fait avant l'enregistrement des poids à 8,6 et $\mathbf{1} 2$ semaines respectivement dans les autres cas.

\section{MÉTHODES}

La méthode proposée ici permet de comparer des groupes deux à deux.

Soient deux souches A et B.

Soient $s_{1 \mathrm{~A}}^{2}, s_{2 \mathrm{~A}}^{2}$ respectivement la variance observée des $\delta^{*} \delta^{*}$ et des $q 9$ pour un caractère donné dans la souche $A$, ces variances étant basées respectivement sur $\nu_{1 \mathrm{~A}}$ et $\nu_{2 \mathrm{~A}}$ degrés de liberté, et de même avec l'indice B pour la souche $B$.

Soit $f$ la vraie valeur (dans la population supposée infinie) du rapport de la variance des $\delta \delta$ à celle des 우, $f$ étant supposé le même pour les deux souches.

Le rapport $F_{\mathrm{A}}=\frac{s_{1 \mathrm{~A}}^{2}}{f s_{2 \mathrm{~A}}^{2}}$ suit une loi de Fisher-Snedecor à $\nu_{1 \mathrm{~A}}$ et $\nu_{2 \mathrm{~A}}$ degrés de liberté. Si $v_{1 \mathrm{~A}}$ et $\nu_{2 A}$ sont grands, la quantité $Z_{A}=\frac{I}{2} L\left(F_{A}\right)$ est à peu près distribuée normalement (KENDALL, I964) avec les moments :

$$
\begin{aligned}
\mathrm{M}_{\mathrm{A}} & =\frac{\mathrm{I}}{2}\left(\frac{\mathrm{I}}{V_{2 \mathrm{~A}}}-\frac{\mathrm{I}}{V_{1 \mathrm{~A}}}\right) \\
\sigma_{\mathrm{A}} & =\frac{\mathrm{I}}{2}\left(\frac{\mathrm{I}}{V_{1 \mathrm{~A}}}+\frac{\mathrm{I}}{V_{2 \mathrm{~A}}}\right)
\end{aligned}
$$

$Z_{\mathrm{A}}$ est fonction de $f$, dont la vraie valeur est inconnue; mais $f$ disparaît dans l'expression de $Z_{A}-Z_{B}$ qui est égal à $Z_{A}^{\prime}-Z_{B}^{\prime}$ en appelant $Z_{A}^{\prime}$ et $Z_{B}^{\prime}$ les quantités $\frac{I}{2} L\left(F_{A}^{\prime}\right)$ et $\frac{I}{2} L\left(F_{B}^{\prime}\right)$ avec

$$
\mathrm{F}_{\mathrm{A}}^{\prime}=\frac{s_{1 \mathrm{~A}}^{2}}{s_{2 \mathrm{~A}}^{2}}, \mathrm{~F}_{\mathrm{B}}=\frac{s_{1 \mathrm{~B}}^{2}}{s_{2 \mathrm{~B}}^{2}}
$$

$Z_{\dot{A}}-Z_{B}$ différence de deux variables normales, est une variable normale, et l'expression calculable à partir des données

$$
\frac{Z_{\mathbf{A}}^{\prime}-Z_{\mathbf{B}}^{\prime}-\left(\mathrm{M}_{\mathbf{A}}-\mathrm{M}_{\mathbf{B}}\right)}{\sqrt{\sigma_{\mathrm{A}}^{2}+\sigma_{\mathrm{B}}^{2}}}
$$

est une variable normale réduite.

Sans fournir un test global d'homogénéité du rapport des variances entre sexes pour plus de deux groupes, cette méthode permet certaines conclusions à partir des comparaisons entre groupes pris deux à deux, à condition que dans chacun l'effectif des deux sexes soit assez important.

\section{RÉSULTATS}

Dans les tableaux I et 2 est testée, suivant la méthode ci-dessus, 1'homogénéité du rapport de la variance des femelles à celle des mâles, respectivement parmi les souches de Jouy pour le poids à 8 semaines et parmi les souches "ponte " du Magne-

(1) Concernant la comparaison des poids adultes au Magneraud, les coqs des diverses souches étaient élevés dans des conditions comparables, mais séparément des femelles, depuis ro semaines d'âge. 


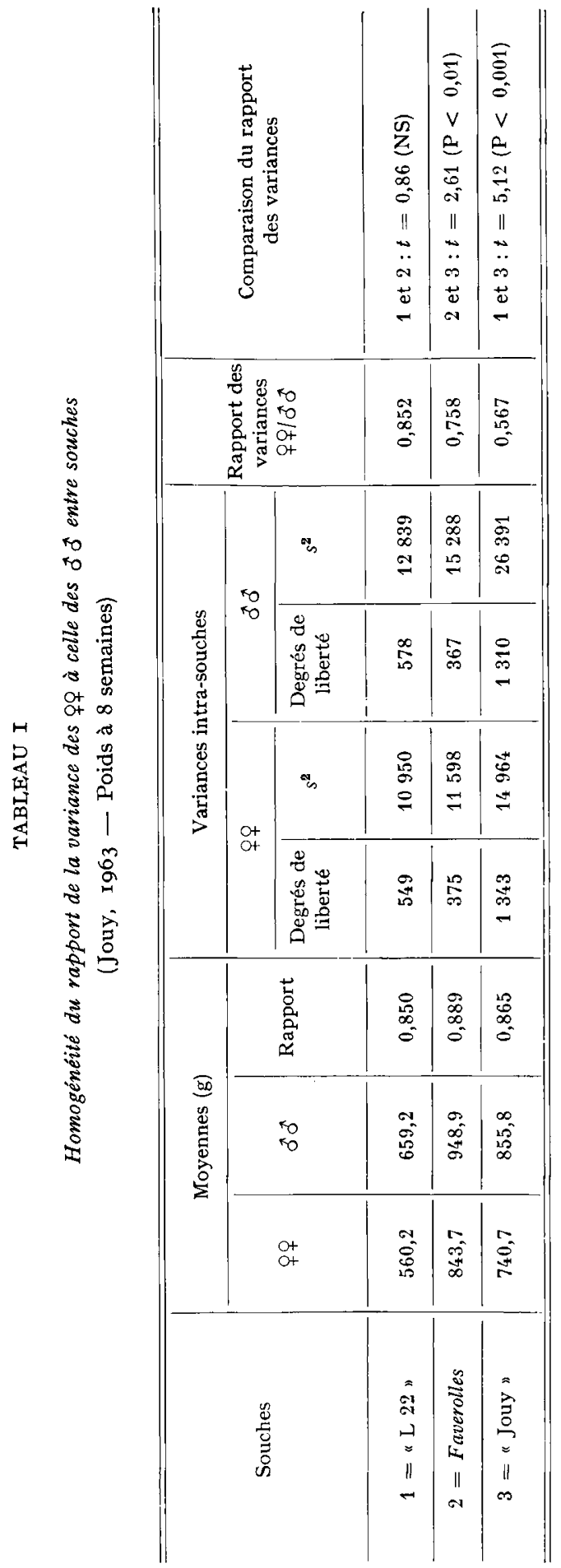




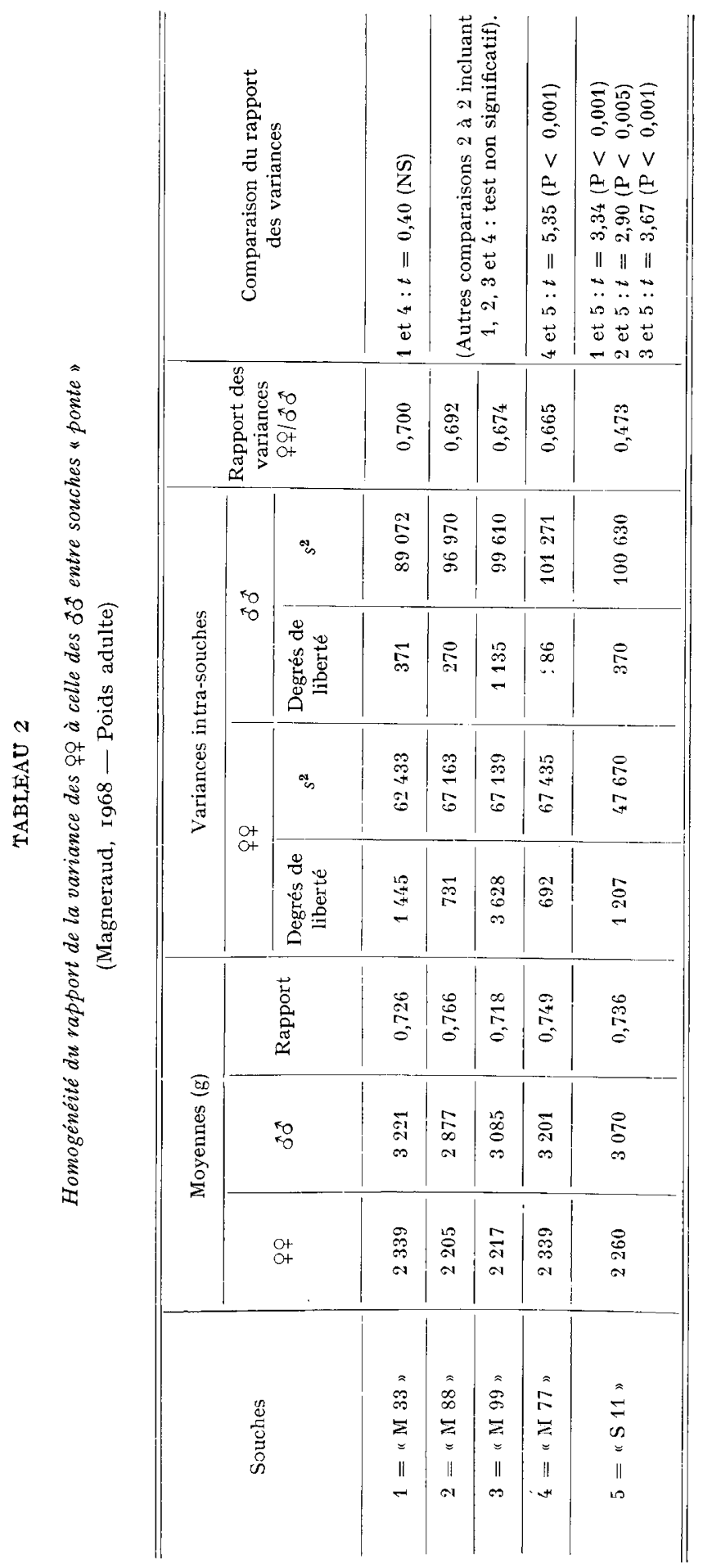




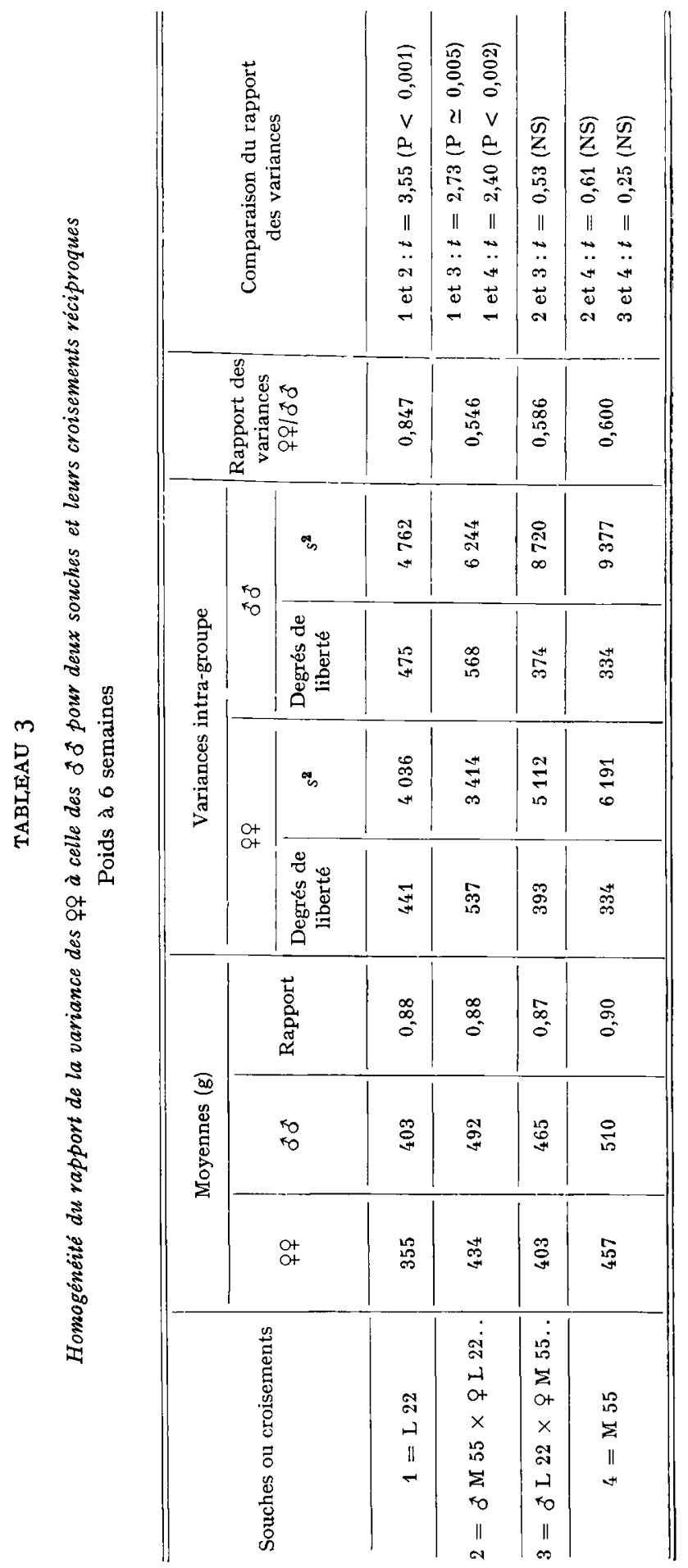




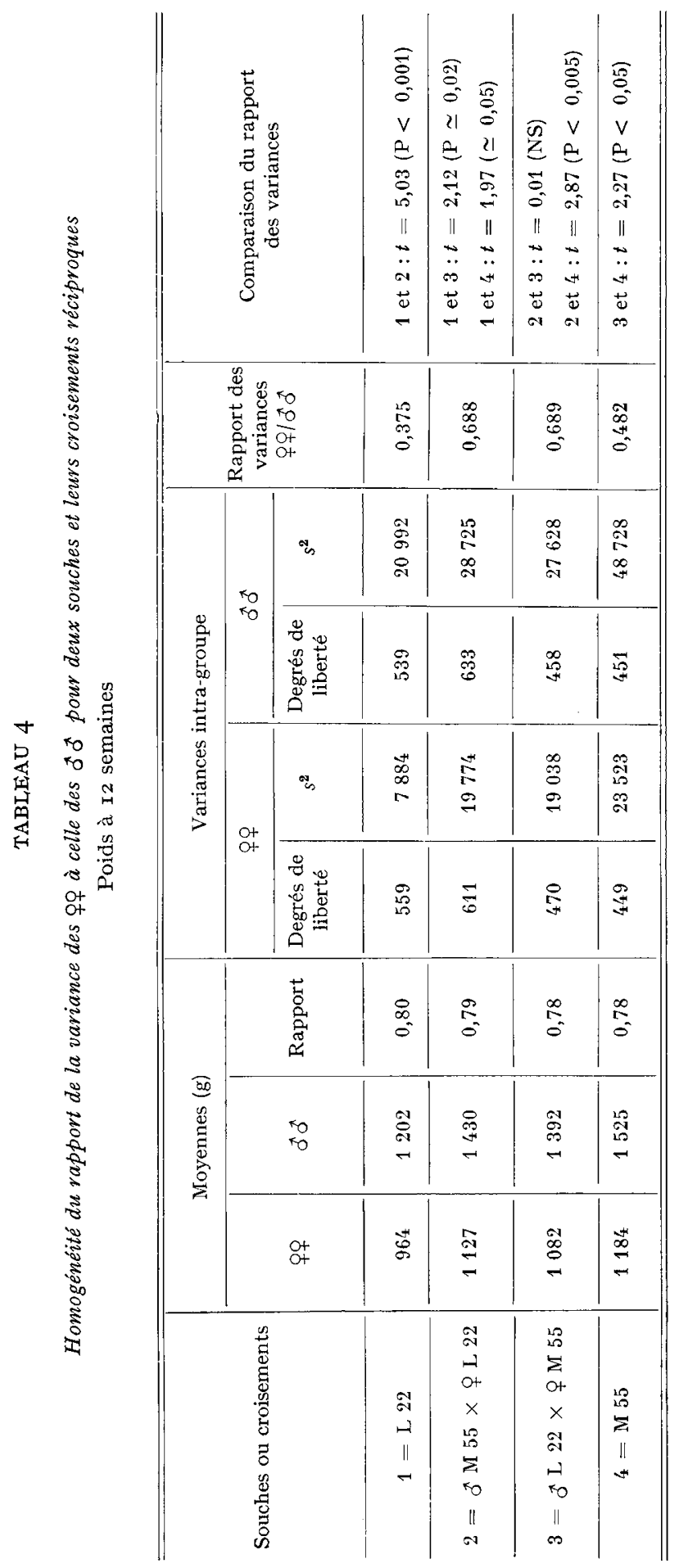


raud pour le poids adulte. Les valeurs moyennes des $\delta^{t} \delta^{t}$ et des $q$ 우 et leur rapport sont indiquées au début de chaque tableau, quoiqu'elles ne soient pas utilisées pour le test présent, et de même pour les variances.

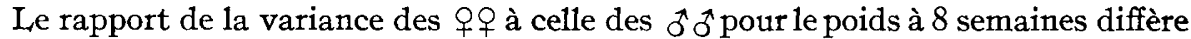
de façon hautement significative entre la souche "Jouy " et les deux autres (tabl. I).

Le tableau 2 montre que, pour le poids adulte, la souche SIr diffère significativement de toutes les autres, les femelles y étant moins variables qu'ailleurs comparativement aux mâles.

Pour les comparaisons entre souches parentales et leurs croisements (données de Nouzilly), on voit (tabl. 3 et 4) que le rapport de la variance des 우우 à celle des $\hat{0} \widehat{o}$ varie entre 0,546 et 0,847 suivant le groupe considéré à l'âge de 6 semaines, et entre 0,375 et 0,689 à I2 semaines. A 6 semaines, les souches pures diffèrent de façon significative au seuil 5 p. Ioo pour le rapport de la variance dans les deux sexes. Par contre, les croisements réciproques ne montrent de différence significative ni entre eux, ni avec le parent $\mathrm{M} 55$.

A I2 semaines, il y a de nouveau une différence significative au seuil $5 \mathrm{p}$. Ioo entre les deux souches parentales. Les deux croisements ne diffèrent pas entre eux, mais ils s'écartent notablement des souches parentales $(\mathrm{P}<0$,00I et $\mathrm{P}<0,005)$.

\section{DISCUSSION ET CONCLUSIONS}

En ce qui concerne le poids adulte, l'hétérogénéité du rapport des variances dans les deux sexes ne peut guère s'expliquer par un tri des mâles sur le début de leur croissance (poids à 8 semaines) plus ou moins sévère suivant la souche. Un tel tri pourrait effectivement influer sur la variance du poids des mâles adultes, du fait de la corrélation existant entre ce caractère et le poids à 8 semaines, mais l'examen du tableau 2 montre que la proportion de mâles éliminés de cette façon ne varie pas de façon très importante suivant la population (valeurs extrêmes 60 et 74 p. Ioo, la souche S II étant intermédiaire).

D'autre part, une différence importante entre souches pour le poids moyen pourrait a priori apporter une perturbation dans les variances dans l'hypothèse d'une corrélation entre moyennes et variances. Toutefois, pour le poids à 8 semaines, la souche "Jouy ", différente des deux autres pour le rapport de la variance dans les deux sexes, est intermédiaire entre elles pour le poids moyen. Il en est de même, dans le cas du poids adulte, pour la souche S Ir vis-à-vis des autres. On pourrait, à la rigueur, se poser une question, relativement aux poids à 6 semaines, pour la souche L 22, différente des autres à la fois pour sa valeur moyenne et pour la variance comparée des deux sexes, mais ce rapport des variances se classe en sens inverse par rapport aux autres souches à $\mathrm{I} 2$ semaines.

On n'observe pas non plus, dans l'ensemble, de relation nette entre rapport de la variance dans les deux sexes et ordre de grandeur global de cette variance, sexes groupés. Seule, à 8 semaines, la souche " Jouy " est, dans les deux sexes, un peu plus variable que les autres.

Nos résultats suggèrent donc que les différences constatées relatives à la variance comparée du poids dans les deux sexes ont une cause biologique spécifique. Les 
souches étudiées étant placées, pour chaque comparaison, dans des conditions d'élevage aussi comparables que possible (spécialement pour les données de Nouzilly), on peut penser à un déterminisme d'origine génétique. Il est impossible de l'analyser plus avant, plusieurs hypothèses étant $a$ priori acceptables :

- Gènes à manifestation différente suivant le sexe, autosomaux ou non, variables suivant la souche.

- Variabilité différente entre souches relativement à des gènes liés aux sexe.

- Différence entre souches pour le degré d'homozygotie des gènes liés au sexe, dans la mesure où ce dernier modifierait la variance du poids des mâles en réponse à l'environnement, comme c'est souvent le cas pour le taux de consanguinité en général (LERNER, I954).

La dernière hypothèse paraît peu vraisemblable, aucune des souches comparées n'ayant un degré très élevé de consanguinité. Quant à la première, on peut rappeler que des gènes identifiés ont parfois été trouvés associés à un effet différentiel sur la variance dans les deux sexes. Ainsi, plusieurs chercheurs ont noté une variance du poids à 8 ou ro semaines plus grande chez les mâles porteurs du gène $K$ d'emplumement lent que pour les mâles à emplumement rapide, sans qu'il y ait de différence appréciable chez les femelles (GLAZENER et JULL, I946 ; GODFREY et FARNSWORTh, I952 ; HURRy et NORdSKog, I953; GoodMan et MUIR, I965; MÉrat, I967).

En fin de compte, il serait intéressant de vérifier si l'on retrouve fréquemment, dans d'autres cas, des différences appréciables entre populations élevées dans les mêmes conditions, pour la variabilité comparée des deux sexes. D'un point de vue pratique, ceci pourrait avoir un certain intérêt pour le poids à l'âge de l'abattage dans des souches ou croisements " chair ».

Reçu pour publication en octobre 1972.

\section{REMERCIEMENTS}

Nous remercions MM. J.-P. Boyer, Station de Recherches avicoles, 37 - Nouzilly, et L.-P. Cochez, Station expérimentale d'Aviculture du Magneraud, d'avoir mis à notre disposition les données qui ont permis le présent travail.

\section{SUMMARY}

VARIATION BETWEEN STRAINS FOR THE RATIO

OF FEMALE TO MALE BODY WEIGHT VARIANCE IN THE FOWL,

Differences in the ratio of female to male body weight variance at various ages were observed between several poultry strains bred in the same conditions and having average weights of the same order. A genetic origin is suggested for these differences. 


\section{RÉFÉRENCES BIBLIOGRAPHIQUES}

Ayoub H., Mérat P., 1972. Différences entre familles pour le dimorphisme sexuel du poids à un âge donné chez la poule. Ann. Génét. Sél. anim., 4, 45-59.

Glazener E. W., JUll M. A., I946. Rate of feathering and ro week body weight observations in strains differing in shank length. Poult. Sci., 25, 433-439.

Godfrey G. F., Farnsworth G. M. Jr., I952. Relation of the sex linked rapid feathering gene to chick growth and mortality. Poult. Sci., 31, 65-68.

Goodman B. L., Muir F. V., r 965 . The influence of comb and feathering phenotypes on body weight and dressing percentage. Poultr. Sci., 44, 644-648.

HurRy H. F., Nordskog A. W., 1953. A genetic analysis of chick feathering and its influence on growth rate. Poultr. Sci., 32, 18-25.

KENDALL M. G., I964. The advanced theory of statistics. II. Griffing and Co, London.

LeRner J. M., I954. Genetic homeostasis. Oliver and Boyd, Edinburgh.

MÉrat P., I967. Contribution à l'étude de la "valeur sélective " associée à quelques gènes chez la poule domestique. I. différences quantitatives liées au génotype individuel. Ann. Biol. anim. Bioch. Biophys., 7, 79-104. 